\title{
Controle de qualidade farmacêutico de comprimidos revestidos provenientes de queixas técnicas associadas ao aspecto do produto
}

\author{
Pharmaceutical quality control of film-coated tablets \\ associated with product's aspect deviation reports
}

\author{
Jaqueline Kalleian Eserian
}

Como citar este artigo: ESERIAN, JAQUELINE K.; Controle de qualidade farmacêutico de comprimidos revestidos provenientes de queixas técnicas associadas ao aspecto do produto. Revista Saúde (Sta. Maria). 2020; 46 (2)

\section{Autor correspondente: Nome: Jaqueline Kalleian Eserian E-mail: jaqueline.eserian@ial. sp.gov.br \\ Telefone: ( 1 l 3068-2930 \\ Formação Profissional: Formada em Farmácia pelas Faculdades Oswaldo Cruz (FOC) que fica na cidade de São Paulo, SP, Brasil. Doutora e mestre em Ciências pela Universidade Federal de São Paulo (UNIFESP), São Paulo, SP, Brasil. \\ Filiação Institucional: Pesquisador Científico. Centro de Medica- mentos, Cosméticos e Saneantes, Instituto Adolfo Lutz, São Paulo, SP, Brasil. \\ Endereço para correspondencia: Avenida Doutor Arnaldo n³55, \\ Prédio $B Q, 5^{\circ}$ andar \\ Bairro: Cerqueira César \\ Cidade: São Paulo \\ Estado: São Paulo \\ CEP: 01246-902}

Data de Submissão:

$21 / 05 / 2020$

Data de aceite:

30/10/2020

Conflito de Interesse: Não há conflito de interesse

\section{(cc) $\mathrm{BY}-\mathrm{NC}-\mathrm{ND}$}

\section{RESUMO}

Objetivo: Verificar a qualidade de comprimidos revestidos provenientes de notificações de queixas técnicas associadas ao aspecto encaminhadas por serviços de vigilância sanitária do estado de São Paulo entre os anos de 2012 e 2017. Métodos: Análises fiscais e de orientação foram realizadas em seis lotes de comprimidos revestidos contendo a substância cloridrato de ranitidina provenientes de três fabricantes diferentes, incluindo medicamentos genéricos e similares. Foram executados ensaios de aspecto, variação de peso, identificação e teor da substância ativa, uniformidade de doses unitárias e dissolução. Resultados: Foram constatadas não conformidades no aspecto de duas dentre as seis amostras analisadas, tais como presença de rachaduras no revestimento dos comprimidos, problemas na embalagem primária (informações impressas sem nitidez suficiente, enrugamento do blister e falta de aderência entre a folha de alumínio laminado e a cartela plástica base) e alteração da cor do comprimido (de branco para amarelado). Desvios da qualidade associados ao aspecto descaracterizam o produto, alterando sua aparência e comprometendo a aderência ao tratamento, sendo que não conformidades associadas ao revestimento evidenciam problemas relativos à própria tecnologia do produto. Todos os ensaios físico-químicos executados apresentaram resultados satisfatórios. Considerações finais: A investigação laboratorial de queixas técnicas garante que medicamentos que não apresentem padrão satisfatório de qualidade sejam recolhidos do mercado, contribuindo para a qualificação de fornecedores e melhoria da oferta dos produtos.

PALAVRAS-CHAVE: Vigilância sanitária de produtos; Controle de qualidade; Tecnologia farmacêutica.

\section{ABSTRACT}

Objective: To evaluate the quality of film-coated tablets associated with reports of aspect problems sent by surveillance vigilance services of São Paulo between 2012 and 2017. Methods: Fiscal and orientation analyses were performed in six batches of coated tablets containing ranitidine hydrochloride from three different manufacturers, including generic and similar drugs. Aspect, weight variation, drug identification and potency, uniformity of dosage units and dissolution tests were performed. Results: Aspect non-compliance was found in two of the six evaluated samples, including cracking in the tablet coating, problems in the primary packaging (lack of clearness in the printed information, blister wrinkling and lack of adherence between the aluminium sheet and plastic base) and color alteration (from white to yellowish). Quality deviations associated with the aspect bring loss of product's characteristics and compromises treatment adherence. Coating non-compliances reflect problems associated with the product's technology. All physico-chemical assays were satisfactory. Final considerations: Laboratorial investigation of product quality problems ensures that drugs that do not comply with quality standards are taken off the market, contributing for supplier qualification and availability of better quality products.

KEYWORDS: Health surveillance of products; Quality control; Technology, pharmaceutical. 


\section{INTRODUÇÃO}

Comprimidos revestidos têm sido utilizados pela indústria farmacêutica por mais de trinta anos, sendo bastante frequente sua aplicação nos dias atuais. Revestimentos podem ser classificados em não-funcionais, também chamados de convencionais, e funcionais. $O$ revestimento funcional é aquele que modifica a liberação da substância ativa contida no comprimido ${ }^{1-3}$.

Já a utilização de revestimento não funcional objetiva a melhora da estabilidade da substância ativa contra agentes externos, como ar, umidade e luz, a mudança da aparência do produto em relação à textura e cor, e o mascaramento de características organolépticas desagradáveis, entre outros ${ }^{1,4}$.

Alterações no aspecto de comprimidos podem levar à notificação de queixas técnicas por parte da equipe de saúde ou mesmo por pacientes, uma vez que desvios de qualidade associados ao aspecto inviabilizam a administração do medicamento, comprometendo o tratamento farmacológico.

Define-se queixa técnica como "qualquer notificação de suspeita de alteração ou irregularidade de um produto ou empresa, relacionada a aspectos técnicos ou legais, e que poderá ou não causar dano à saúde individual ou coletiva"5. Os serviços de vigilância sanitária são responsáveis por avaliar as notificações, classificando os riscos sanitários e planejando as ações a serem tomadas, tais como inspeção do fabricante, coleta de amostras para análise fiscal ou até mesmo a adoção de medidas preventivas ${ }^{6}$.

As análises fiscais em medicamentos incluem a realização de ensaios físico-químicos a fim de se verificar a qualidade do produto. No caso de formas farmacêuticas sólidas, tais como comprimidos revestidos, realizam-se ensaios relacionados à presença/ ausência da substância ativa (ensaio de identificação), potência da substância ativa (ensaio de teor), variação na quantidade de substância ativa entre as doses (ensaio de uniformidade de doses) e liberação da substância ativa a partir da forma farmacêutica (ensaio de dissolução), além da avaliação do aspecto do produto. Resultados insatisfatórios em qualquer um dos ensaios mencionados evidenciam desvios de qualidade do medicamento, resultando em ações de vigilância sanitária.

O objetivo deste estudo foi verificar a qualidade de comprimidos revestidos provenientes de notificações de queixas técnicas associadas ao aspecto encaminhadas por serviços de vigilância sanitária do estado de São Paulo entre os anos de 2012 e 2017.

\section{MÉTODO}

\section{Amostras}

Foram analisados comprimidos revestidos contendo a substância cloridrato de ranitidina $150 \mathrm{mg}$ provenientes 
de três fabricantes diferentes ( $A, B$ e $C$ ), incluindo medicamentos genéricos e similares. Foram avaliados um lote do Fabricante A, três lotes do Fabricante B (denominados B1, B2 e B3) e dois lotes do Fabricante C (denominados C1 e C2). As amostras foram colhidas pelos serviços de vigilância sanitária locais e encaminhadas para o Laboratório Central de Saúde Pública do Estado de São Paulo para análise.

\section{Ensaios}

- Aspecto

Realizado por inspeção visual do material. Foram avaliados o acondicionamento da amostra (originalidade e lacre da embalagem) e a presença de não conformidades, de acordo com o previsto no registro do medicamento/ bula.

- Variação de peso

Determinou-se o peso médio de 20 comprimidos em balança analítica (Mettler Toledo, AL204, Columbus, EUA). Tolera-se até dois comprimidos fora do limite de $\pm 5 \%$ em relação ao peso médio, mas nenhum comprimido fora do dobro deste limite $( \pm 10 \%)^{7}$.

- Identificação e teor da substância ativa

Pulverizou-se os 20 comprimidos utilizados no ensaio anterior. Transferiu-se quantidade de pó equivalente a um peso médio para balão volumétrico de $250 \mathrm{~mL}$. Adicionou-se água destilada (Elgastat Maxima Veolia, Paris, França) até metade do volume do balão, sonicou-se por 15 minutos e agitou-se mecanicamente por mais 15 minutos. Tal solução foi filtrada e diluída sucessivamente no mesmo solvente até concentração teórica de 12,5 $\mathrm{gg} / \mathrm{mL}$.

A solução padrão foi preparada utilizando-se padrão primário de cloridrato de ranitidina (INCQS, Rio de Janeiro, Brasil). Preparou-se uma curva de calibração $(5,10,12,5$ e 15 $\mathrm{gg} / \mathrm{mL})$ com o mesmo solvente. As análises foram realizadas em um espectrofotômetro UV-Vis HP 8453 (HP/ Agilent, Santa Clara, EUA). A identificação da substância ativa é feita por meio da comparação dos espectros de absorção no UV obtidos a partir da leitura das soluções amostra e padrão, de 200 a $400 \mathrm{~nm}$.

O teor da substância ativa é calculado a partir da obtenção dos valores de absorbância das soluções amostra e padrão em 314nm. Em ambos os ensaios utilizou-se água destilada como branco. O produto deve apresentar identificação positiva para cloridrato de ranitidina e teor entre 90 e 110\% da quantidade declarada de substância ativa no rótulo para ser aprovado?.

- Uniformidade de doses unitárias

Realizado por meio de variação de peso de 10 comprimidos. A quantidade de substância ativa por comprimido é estimada a partir do resultado do ensaio de teor e dos pesos individuais dos comprimidos. O valor de aceitação (VA), calculado de acordo com método farmacopeico, deve apresentar resultado menor que 15. Caso o VA seja maior que 15, outros 20 comprimidos devem ser testados. Para o total de 30 comprimidos, o VA deve ser menor ou igual a 15 e nenhum 
comprimido deve apresentar quantidade de substância ativa fora do intervalo de 75 a $125 \%$ da quantidade declarada .

- Dissolução

Seis comprimidos foram testados em cubas individuais em aparelho dissolutor Erweka DT 12R (Erweka, Heusenstamm, Alemanha). Utilizou-se $900 \mathrm{~mL}$ de $\mathrm{HCl} 0,1 \mathrm{M}\left(37 \pm 0,5^{\circ} \mathrm{C}\right)$ como meio de dissolução, aparato 2 (pás) e velocidade de agitação de 50rpm por 45 minutos. Após o teste, alíquotas de $5 \mathrm{~mL}$ foram transferidas para balões volumétricos de $100 \mathrm{~mL}$ e diluídas em HCl 0,1M, obtendo-se soluções com concentração teórica de $8,3 \mu \mathrm{g} / \mathrm{mL}$. Conforme método do fabricante, as absorbâncias das soluções amostra e da solução padrão, preparada na mesma concentração e com o mesmo solvente, foram medidas em $224 \mathrm{~nm}$. Cada comprimido deve liberar no mínimo $Q(80 \%)+5 \%$ do valor declarado de substância ativa no tempo estipulado, de acordo com especificação do fabricante.

\section{RESULTADO}

O ensaio de aspecto de medicamentos é um ensaio relativamente simples, porém bastante importante. A Tabela 1 apresenta um resumo das queixas técnicas avaliadas e os respectivos resultados do ensaio de aspecto.

Tabela 1. Resumo das queixas técnicas avaliadas e respectivos resultados do ensaio de aspecto nos comprimidos revestidos.

\begin{tabular}{llll}
\hline Amostra & Tipo de análise & Queixa técnica & Ensaio de aspecto \\
\hline A & Fiscal & Comprimidos com rachaduras & Não conforme \\
B1 & Fiscal & Alteração de cor & Conforme \\
B2 & Fiscal & Alteração de cor & Conforme \\
B3 & Fiscal & Alteração de cor & Conforme \\
C1 & Orientação & Suspeita de degradação devido à alteração de cor & Não conforme \\
C2 & Fiscal & Suspeita de degradação devido à alteração de cor & Conforme \\
\hline
\end{tabular}

Verificou-se que os comprimidos do Fabricante Aapresentavam rachaduras/cracking em grande extensão, sendo possível visualizar tal não conformidade quando os comprimidos estavam ainda acondicionados nos alvéolos do blister. Devido às rachaduras/cracking, alguns comprimidos partiram-se e esfarelaram-se quando foram retirados dos alvéolos. Além disso, foi possível verificar não conformidades na embalagem primária, tal como informações impressas sem nitidez suficiente, enrugamento do blister e falta de aderência entre a folha de alumínio laminado e a cartela plástica base.

Para as três amostras do Fabricante B (B1, B2 e B3), verificou-se que a queixa de alteração de cor (de branco para amarelo) não procedia. O fato é que a aparência amarelada dos comprimidos era percebida somente quando os 
blisteres encontravam-se armazenados na embalagem secundária (caixa de cartolina/ embalagem hospitalar) devido ao reflexo da faixa amarela que os identifica como genérico impressa no blister adjacente. Desta forma, não houve não conformidade no aspecto dos comprimidos.

Uma das amostras do Fabricante C (C1) encontrava-se violada, sendo colhida diretamente do paciente reclamante. Tal amostra apresentava embalagem secundária (cartucho de cartolina) violada, um blister com quatro comprimidos armazenados em alvéolos inviolados com aspecto satisfatório (comprimidos brancos) e um comprimido fragmentado com alteração de cor (de branco para amarelado) em alvéolo violado fechado com fita adesiva incolor. A queixa indicava suspeita de degradação dos comprimidos devido à alteração de cor percebida em uma das unidades do blister pelo próprio reclamante. Para esta amostra, foi realizada uma análise de orientação devido às condições de sua colheita.

A amostra C2 foi colhida, então, para fins de análise fiscal frente à suspeita de desvio de qualidade evidenciada na análise de orientação realizada na amostra C1. Entretanto, tal amostra pertencia a um lote diferente do lote da amostra C1. Esta amostra estava inviolada, apresentando aspecto satisfatório.

Ainda, foram realizados ensaios de variação de peso, identificação e teor de substância ativa e uniformidade de doses unitárias nas amostras dos Fabricantes B e C. Devido ao grande comprometimento no aspecto dos comprimidos do Fabricante A, tornou-se inviável a realização de ensaios físico-químicos nesta amostra. 0 espectro de absorção no ultravioleta obtido no ensaio de identificação da substância ativa e a curva de calibração utilizada para sua quantificação são apresentados nas Figuras 1 e 2, respectivamente.

Figura 1. Espectro de absorção no ultravioleta, exemplificado por meio da análise das amostras C1 (vermelho) e C2 (verde) frente ao padrão de cloridrato de ranitidina (azul marinho).

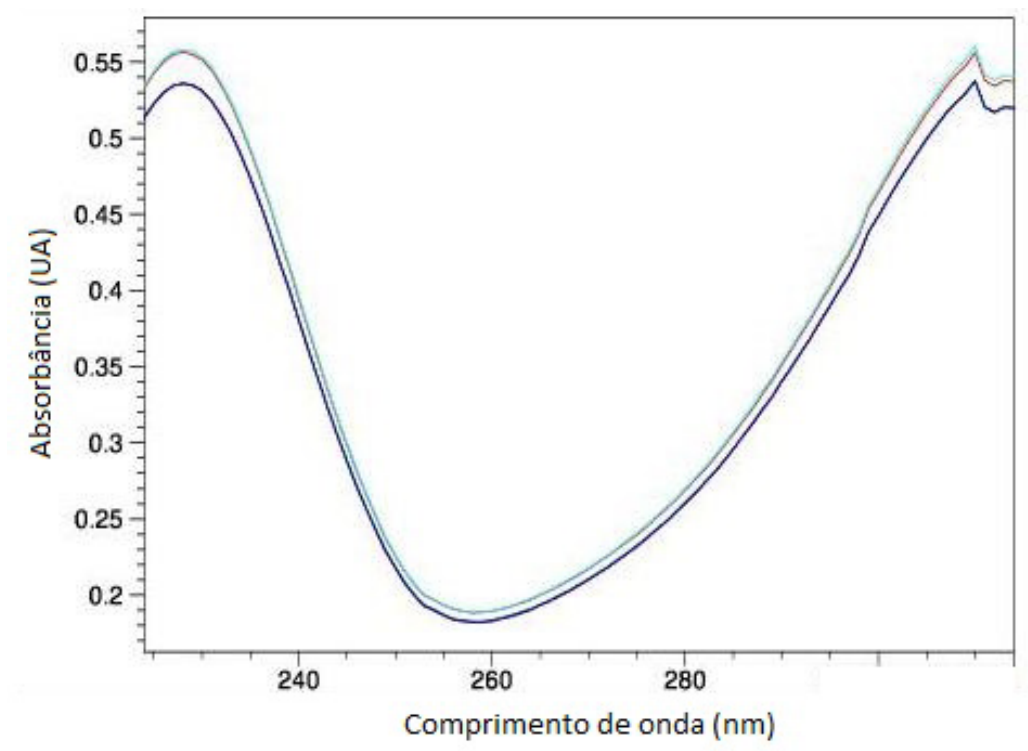


Figura 2. Curva de calibração de cloridrato de ranitidina obtida por espectroscopia no ultravioleta (concentrações utilizadas: $5,10,12,5$ e $15 \mu \mathrm{g} / \mathrm{mL})$.

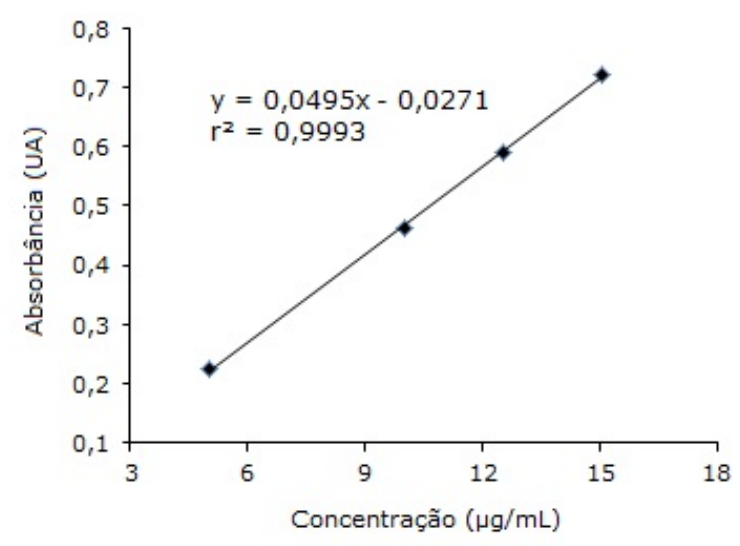

O ensaio de dissolução foi realizado somente para as amostras do Fabricante B devido à quantidade de amostras do Fabricante $\mathrm{C}$ ser insuficiente, priorizando-se então para estas amostras os ensaios mais relacionados à queixa de suspeita de degradação do produto (identificação e teor de substância ativa). Para a amostra C1, realizou-se os ensaios de identificação e teor de substância ativa em um pool de três comprimidos armazenados em alvéolos inviolados, devido à quantidade de amostra disponível.

Todos os ensaios físico-químicos realizados apresentaram resultados satisfatórios (Tabela 2).

Tabela 2. Resultados dos ensaios físico-químicos provenientes de queixas técnicas de suspeita de desvio de qualidade relacionadas ao aspecto em comprimidos revestidos.

\begin{tabular}{cccccc}
\hline $\begin{array}{c}\text { Amostral } \\
\text { Ensaio }\end{array}$ & $\begin{array}{c}\text { Variação } \\
\text { de peso }\end{array}$ & $\begin{array}{c}\text { Identificação de } \\
\text { substância ativa }\end{array}$ & $\begin{array}{c}\text { Teor de } \\
\text { substância ativa }\end{array}$ & $\begin{array}{c}\text { Uniformidade } \\
\text { de doses } \\
\text { unitárias (VA) }\end{array}$ & Dissolução \\
\hline $\mathrm{A}^{*}$ & - & - & - & - & - \\
B1 & $-2,3 \%$ & Positiva & $95,7 \%$ & 5,8 & $>85 \%$ \\
& $+2,8 \%$ & & & & \\
B2 & $-2,8 \%$ & Positiva & $93,0 \%$ & 9,8 & $>85 \%$ \\
& $+3,3 \%$ & & & & \\
B3 & $-2,9 \%$ & Positiva & $94,5 \%$ & 8,0 & $>85 \%$ \\
& $+4,1 \%$ & & & & - \\
C1 $^{z}$ & - & Positiva & $94,1 \%{ }^{\#}$ & - & - \\
$\mathrm{C}^{z}$ & $-1,67 \%$ & Positiva & $92,0 \%$ & 8,5 & \\
& $+1,63 \%$ & & & & \\
\hline
\end{tabular}

${ }^{*}$ Comprimidos com aspecto muito comprometido para realização de ensaios físico-químicos; ${ }^{2}$ Devido à quantidade insuficiente de amostra, foram priorizados os ensaios relacionados à queixa. \#Ensaio realizado em pool de três comprimidos. 


\section{DISCUSSÃO}

Embora as queixas fossem relacionadas ao aspecto dos medicamentos, diversos ensaios físico-químicos foram realizados nas amostras a fim de se obter uma visão mais ampla da qualidade dos produtos notificados.

Os ensaios de identificação e teor são frequentemente realizados juntos e visam atestar, por meio de amostragem, a presença e a potência da substância ativa no lote, respectivamente. Já o ensaio de uniformidade de doses unitárias evidencia a quantidade de substância ativa por comprimido, sendo essencial para a verificação da precisão entre as doses que serão administradas. 0 ensaio de dissolução, por sua vez, é um ensaio in vitro que auxilia na previsão do desempenho do produto in vivo.

Desta forma, entende-se que a realização de ensaios físico-químicos contribui de maneira essencial para a garantia da qualidade do medicamento e consequente eficácia e segurança do tratamento. Todos os ensaios físicoquímicos realizados apresentaram resultados satisfatórios, embora tenham sido realizados em sua totalidade apenas para as amostras B1, B2 e B3, conforme salientado anteriormente.

As notificações de queixas técnicas possibilitam o estabelecimento do perfil das ocorrências, qualificando a assistência de um modo geral tanto em relação a melhorias na qualidade do produto quanto à retirada de lotes não conformes do mercado $0^{6,8}$.

Os problemas de aspecto evidenciados neste estudo parecem ser comuns aos observados em outros cenários do serviço de saúde do país. Uma revisão da literatura apontou que a maior parte das notificações realizadas por hospitais do país envolvendo medicamentos eram relacionadas a problemas que podiam ser caracterizados visualmente antes mesmo de sua utilização, tal como alterações no aspecto, falhas na embalagem, discrepância na quantidade de produto disponibilizado e erros em informações do rótulo6,8-10.

Lima et al. ${ }^{9}$, em um estudo realizado em um hospital sentinela sobre queixas técnicas e eventos adversos a medicamentos, apontou que, num período de dois anos, 199 notificações foram feitas ao setor de farmacovigilância do hospital. Dentre essas, $70 \%$ eram relacionadas a queixas técnicas (desvios de qualidade), $21 \%$ à ineficácia terapêutica e $9 \%$ a reações adversas a medicamentos ${ }^{9}$.

As queixas técnicas mais notificadas foram vazamento $(17,3 \%)$ e coloração diferente do habitual $(10,8 \%)$, mencionando-se ainda aspecto diferente do habitual $(5,0 \%)$ e rótulo de difícil identificação $(4,3 \%)^{9}$.

Os hospitais sentinela atuam de forma articulada com a vigilância sanitária em relação à notificação de eventos adversos e queixas técnicas de produtos utilizados na atenção à saúde ${ }^{5}$. Caon et al. ${ }^{8}$ mostrou que de 169 notificações realizadas num período de um ano em um outro hospital sentinela, aproximadamente $65 \%$ também estavam relacionadas a queixas técnicas de medicamentos. Dentre essas, $54,1 \%$ eram relativas a problemas na embalagem e $21,1 \%$ ao 
aspecto do produto (alteração de cor, cristalização ou presença de corpo estranho) ${ }^{8}$.

Desvios da qualidade associados ao aspecto acabam por descaracterizar o produto, alterando sua aparência e consequentemente comprometendo a aderência ao tratamento ${ }^{1}$. Não conformidades associadas ao revestimento de comprimidos evidenciam problemas relativos à própria tecnologia do produto.

As não conformidades em comprimidos revestidos podem estar associadas ao estresse gerado na etapa de secagem do revestimento, ainda durante a fabricação do produto. Autilização de temperaturas maiores que a temperatura ambiente durante essa etapa pode afetar a força, elasticidade e adesão do revestimento. À medida que os comprimidos revestidos retornam à temperatura ambiente, um estresse térmico é formado, contribuindo para o surgimento de rachaduras/cracking ${ }^{1,4}$.

Já a variação de cor do revestimento pode estar associada a uma homogeneização ineficiente ou a alterações das condições de pulverização do revestimento, tais como frequência e duração da pulverização e tamanho e formato da zona de pulverização".

Evidências apontam maior risco de não conformidades em geral no revestimento de comprimidos que são mais curvos, quando comparados àqueles que são planos $^{1,11}$. Cabe destacar que os comprimidos de todos os fabricantes analisados eram biconvexos, ou seja, apresentavam certo grau de curvatura.

Este estudo apresenta algumas limitações, como a quantidade insuficiente de comprimidos encaminhados com as amostras $\mathrm{C} 1$ e C2, fato que inviabilizou a realização de todos os ensaios físico-químicos. A falta de detalhes sobre as queixas técnicas também acabou por limitar a discussão dos casos sob o ponto de vista da atenção farmacêutica.

A notificação de queixas técnicas se qualifica como um dos instrumentos de vigilância sanitária disponíveis para o monitoramento da qualidade dos medicamentos disponíveis no mercado ${ }^{12}$. Além disso, estudos que remetem à vigilância pós-comercialização de medicamentos contribuem com o sistema de vigilância sanitária ao fornecer informações essenciais para a tomada de decisões neste âmbito 9 .

O fornecimento de dados técnico-científicos resultantes de análises de medicamentos, tal como apresentado neste estudo, fortalece as ações de vigilância sanitária e contribui para o sistema público de saúde do país.

\section{CONSIDERAÇÕES FINAIS}

A verificação do aspecto de medicamentos é o único parâmetro para avaliação da qualidade do produto fora do ambiente laboratorial ao alcance de profissionais de saúde e pacientes, podendo ser colocada em prática ainda durante a dispensação ou administração do mesmo. $O$ item aspecto caracteriza-se como uma das principais causas de notificação de queixas técnicas. Embora um aspecto satisfatório não garanta a eficácia e segurança do medicamento, é um ponto 
essencial da qualidade global do produto.

Este estudo apontou que duas dentre as seis amostras de comprimidos revestidos provenientes de queixas técnicas apresentaram resultados não conformes quanto ao aspecto. A investigação laboratorial de queixas técnicas garante que medicamentos que não apresentem padrão satisfatório de qualidade sejam recolhidos do mercado, contribuindo para a qualificação de fornecedores e melhoria da oferta dos produtos.

\section{REFERÊNCIAS}

1. Eserian JK, Lombardo M. Comprimidos revestidos por película: Tipos de não-conformidades e suas causas. Rev Eletronica Farm. 2014;XI(3):32-47.

2. Bikiaris D, Koutri I, Alexiadis D, Damtsios A, Karagiannis G. Real time and non-destructive analysis of tablet coating thickness using acoustic microscopy and infrared diffuse reflectance spectroscopy. Int J Pharm 2012;438(1-2):3344.

3. Kucera SA, Felton LA, McGinity JW. Physical aging in pharmaceutical polymers and the effect on solid oral dosage form stability. Int J Pharm. 2013;457(2):428-36.

4. Gibson SHM, Rowe RC, White ETF. Mechanical properties of pigmented tabletcoating formulations and their resistance to cracking I. Static mechanical measurement. Int J Pharm. 1988;48(1-3):63-77.

5. RDC n 51, de 29 de setembro de 2014. Dispõe sobre a Rede Sentinela para o Sistema Nacional de Vigilância Sanitária. Diário Oficial da União 2014; 01 de outubro.

6. Lombardo M, Eserian JK. A análise da qualidade de medicamentos e o papel do laboratório oficial no contexto da saúde pública. Rev Adm Saúde. 2017;17(67):1-14.

7. Farmacopeia Brasileira. Agência Nacional de Vigilância Sanitária, 5ed. Brasília: Anvisa; 2010.

8. Caon S, Feiden IR, Santos MA. Desvios de qualidade de medicamentos em ambiente hospitalar: identificação 
e avaliação das ocorrências. Rev Bras Farm Hosp Serv Saúde. 2012;3(1):23-6.

9. Lima PF, Cavassini ACM, Silva FAT, Kron MR, Gonçalves SF, Spadotto A, et al. Queixas técnicas e eventos adversos a medicamentos notificados em um hospital sentinela do interior de São Paulo, 2009-2010. Epidemiol Serv Saúde. 2013;22(4):679-86.

10. Azulino ACO, Costa MHA, Carvalho MN, Moreira AS, Oliveira AF, Pinto ACG, et al.. Queixas técnicas realizadas pelos profissionais da saúde, relacionadas aos produtos utilizados em Hospital Sentinela de Belém-Pará. Rev Bras Farm Hosp Serv Saúde. 2013;4(3):13-6.

11. Fassihi AR, Parker MS. Formulation effects on capping tendencies. Int J Pharm. 1986;31(3):271-3.

12. Bezerra ALQ, Camargo e Silva AEB, Branquinho NCSS, Paranaguá TTB. Análise de queixas técnicas e eventos adversos notificados em um hospital sentinela. Rev enferm UERJ. 2009;17(4):467-72. 